\title{
Regimen of 5-Fluorouracil and Cisplatin Increases the Incidence of Extravasation in Patients Undergoing Chemotherapy
}

\author{
HISASHI TAGASHIRA ${ }^{1 *}$, YASUHISA IZUSHI $^{2,3^{*}}$, TOMOKI IKUTA ${ }^{4}$, \\ YASUMASA KOIKE ${ }^{4}$, YOSHIHISA KITAMURA ${ }^{2}$ and HIROSHI YAMAMOTO ${ }^{1}$ \\ ${ }^{1}$ Department of Pharmacy, National Hospital Organization Okayama Medical Centre, Okayama, Japan; \\ ${ }^{2}$ Department of pharmacotherapy, School of Pharmacy, Shujitsu University, Okayama, Japan; \\ ${ }^{3}$ Division of Molecular and Cellular Medicine, Department of Clinical Science, \\ National Hospital Organization Okayama Medical Centre, Okayama, Japan; \\ ${ }^{4}$ Department of Pharmacy, National Hospital Organization Shikoku Cancer Centre, Ehime, Japan
}

\begin{abstract}
Background/Aim: Extravasation associated with chemotherapy can induce localised injury, necrosis, and nerve damage, resulting in discontinued chemotherapy and impaired quality of life; however, risk factors for extravasation remain unclear. The present study aimed to identify chemotherapy regimen-associated factors related to extravasation. Patients and Methods: Data on patient and chemotherapy protocol characteristics were extracted from our hospital's electronic database; the frequency of extravasation was compared among patients receiving different chemotherapy regimens. Results: Twenty-two patients with extravasation undergoing chemotherapy during the study period were enrolled in the present study. Patients undergoing treatment with 5fluorouracil and cisplatin were most likely to develop extravasation in the present study. All patients presenting with extravasation during treatment with 5-fluorouracil and cisplatin developed swelling and many (40\%) developed erythema within the first two cycles of treatment. Conclusion: Treatment with 5-fluorouracil combined with cisplatin increases the incidence of extravasation. Ensuring suitable vascular access and increasing awareness regarding the symptoms and timing of extravasation among patients and medical staff can improve extravasation prevention and diagnosis.
\end{abstract}

This article is freely accessible online.

*These Authors contributed equally to this study.

Correspondence to: Hisashi Tagashira, Department of Pharmacy, National Hospital Organization Okayama Medical Centre, 1711-1 Tamasu, Kita-ku, Okayama 701-1192, Japan. Tel: +81 862949911, e-mail: tagashira.hisashi.je@mail.hosp.go.jp

Key Words: 5-Fluorouracil and cisplatin, extravasation, chemotherapy, regimen.
Extravasation during chemotherapy requires immediate treatment, as it may induce inflammation in subcutaneous tissue and lead to localised injury, necrosis, and nerve damage (1), resulting in discontinued chemotherapy and impaired quality of life (2).

Extravasation is rare, with an incidence ranging from $0.1 \%$ to $6.5 \%$, and the associated risk factors remain unclear and difficult to establish (3). Moreover, ethical restrictions associated with prospective studies and the challenges associated with establishing an animal model of human extravasation have curtailed research progress in this area. The association between chemotherapy regimen and the incidence of extravasation has not been previously studied.

The present study aimed to examine chemotherapy regimen-associated factors related to extravasation, stratified by regimen type. In addition, the present study aimed to identify the characteristics of extravasation among patients undergoing chemotherapy, seeking to support the timely diagnosis and treatment of extravasation.

\section{Patients and Methods}

This study was approved by the Institutional Review Board at the National Hospital Organization Shikoku Cancer Centre (Clinical Study 2019-72) and Okayama Medical Centre (Clinical Study 2019061). Data from patients who underwent chemotherapy at the Shikoku Cancer Centre between April 2012 and April 2013 were extracted from the hospital's database. Patients who developed chemotherapeutic extravasation were eligible for inclusion in the present study. Information on patient characteristics and the circumstances of extravasation was extracted from electronic incidents and medical records. During the study period, chemotherapy and other patient care procedures were administered by a professionally trained medical team that included a physician, nurse, and pharmacist employed by the Department of Oncology.

Assessment of chemotherapy regimen-associated factors related to extravasation. To confirm the association between chemotherapy 
in vivo $35: 1147-1150(2021)$

Table I. Characteristics of patients presenting with chemotherapy-associated extravasation.

\begin{tabular}{|c|c|c|c|c|c|c|c|}
\hline No. & Gender & Cancer & Symptom & Regimen & $\begin{array}{l}\text { Infusion rate } \\
(\mathrm{ml} / \mathrm{h})\end{array}$ & Cycle* & $\begin{array}{l}\text { History: CTx/ } \\
\text { extravasation }\end{array}$ \\
\hline 1 & F & Breast & Induration & PTX & 250 & 13 & $-1-$ \\
\hline 2 & $\mathrm{~F}$ & Breast & Swelling & DTX & 250 & 3 & $+1-$ \\
\hline 3 & $\mathrm{~F}$ & Oesophageal & Swelling, pain & 5-FU-CDDP & 200 & 1 & $+/-$ \\
\hline 4 & M & Oesophageal & Swelling, pain & PTX & 250 & 2 & $+/-$ \\
\hline 5 & $\mathrm{~F}$ & Uterocervical & Swelling, pain, erythema & PTX-CDDP & 250 & 1 & $+/-$ \\
\hline 6 & M & Oesophageal & Swelling & 5-FU-CDDP & 50 & 1 & $-1-$ \\
\hline 7 & M & Oesophageal & Swelling & 5-FU-CDDP & 50 & 2 & $+/+$ \\
\hline 8 & M & Oesophageal & Swelling & 5-FU-CDDP & 50 & 1 & $+/-$ \\
\hline 9 & M & Oesophageal & Swelling & 5-FU-CDDP & 50 & 3 & $-1-$ \\
\hline 10 & M & Oesophageal & Destruction & 5-FU-CDDP & 50 & 2 & $-1-$ \\
\hline 11 & M & Oesophageal & Swelling, pain & PTX & 250 & 2 & $+/+$ \\
\hline 12 & M & Oesophageal & Swelling & 5-FU-CDDP & 50 & 7 & $+/-$ \\
\hline 13 & M & Oesophageal & Swelling, erythema & 5-FU-CDDP & 50 & 3 & $-1-$ \\
\hline 14 & M & Oesophageal & Swelling, erythema & 5-FU-CDDP & 50 & 1 & $-1-$ \\
\hline 15 & $\mathrm{~F}$ & Gastric & Swelling & PTX & 250 & 3 & $-1-$ \\
\hline 16 & $\mathrm{~N}$ & Oesophageal & Swelling, erythema & 5-FU-CDDP & 50 & 2 & $+/+$ \\
\hline 17 & M & Oesophageal & Swelling & 5-FU-CDDP & 50 & 1 & $+/-$ \\
\hline 18 & M & Oesophageal & Swelling, erythema & 5-FU-CDDP & 50 & 5 & $+/-$ \\
\hline 19 & $\mathrm{M}$ & Oesophageal & Swelling, erythema & 5-FU-CDDP & 50 & 2 & $-1-$ \\
\hline 20 & M & Lung & Defective installation & AMR & $500 * *$ & 1 & $+/-$ \\
\hline 21 & $\mathrm{~F}$ & Oesophageal & Swelling, erythema & 5-FU-CDDP & 50 & 2 & $-1-$ \\
\hline 22 & M & Oesophageal & Swelling & 5-FU-CDDP & 50 & 2 & $+/-$ \\
\hline
\end{tabular}

AMR: Amrubicin; CDDP: cisplatin; CTx: chemotherapy; DTX: docetaxel; F: female; 5-FU: 5-fluorouracil; M: male; PTX: paclitaxel. *Appearance of extravasation. $* *$ Maximum infusion rate.

regimen and the change in the frequency of extravasation, we assessed the incidence of extravasation among patients undergoing treatment with each chemotherapy regimen. In addition, we examined the characteristics of the chemotherapy regimen and the symptom profile of patients presenting with extravasation.

Statistical analyses. Comparisons were performed using Fisher's exact test for categorical variables. $p$-Values less than 0.05 were considered indicative of a statistically significant finding. All statistical analyses were performed using GraphPad Prism 8 software (GraphPad Software, San Diego, CA, USA).

\section{Results}

Among 12,475 patients undergoing chemotherapy during the study period, $22(0.18 \%)$ developed chemotherapy-related extravasation and were included in the present study. Patient characteristics are presented in Table I.

Extravasation occurred in patients undergoing treatment with the following regimens: 5-fluorouracil and cisplatin (5FU-CDDP) $(n=15)$, paclitaxel $(n=4)$, paclitaxel and cisplatin $(n=1)$, docetaxel $(n=1)$, and amrubicin $(n=1)$ (Figure 1A). Patients receiving 5-FU-CDDP accounted for $68.2 \%$ of all extravasation cases. The incidence of extravasation in patients undergoing treatment with 5-FU-CDDP was $7.4 \%$, which was significantly higher than that in patients treated with any other regimen (Figure 1B). In addition, treatment with 5-FU-CDDP was administered as a continuous intravenous infusion at $50 \mathrm{ml} / \mathrm{min}$ over $24 \mathrm{~h}$ for 5 days. The details of the infusion protocol for each regimen are presented in Table II.

Eleven $(73.3 \%$ ) patients undergoing treatment with 5-FUCDDP developed extravasation within the first two cycles of chemotherapy (Figure 2).

The most common symptoms of extravasation included swelling, observed in all the patients, and erythema, observed among $40 \%$ of the patients treated with 5-FUCDDP. The proportion of patients experiencing pain was lower than that of patients experiencing swelling and erythema (Figure 3 ).

\section{Discussion}

The present study revealed that the 5-FU-CDDP regimen is associated with a remarkable increased incidence of extravasation in patients undergoing chemotherapy, suggesting that patients being treated with 5-FU-CDDP should be monitored carefully for the signs of extravasation. The administration of the 5-FU-CDDP regimen involves continuous intravenous infusion over the course of 5 days (Table II), which may partly account for the increased incidence of extravasation associated with this treatment (4). 


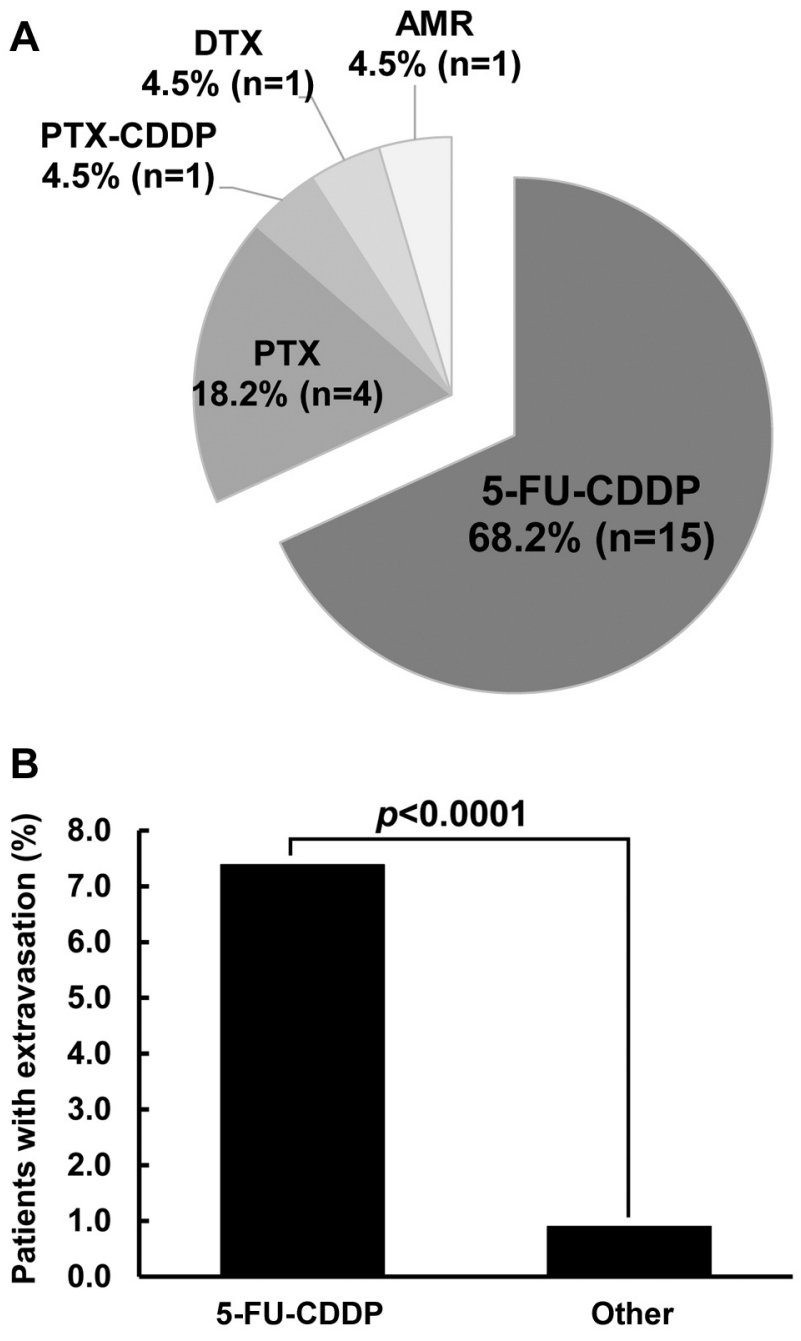

Figure 1. Frequency of chemotherapeutic extravasation. A: Proportion of patients with chemotherapy extravasation during treatment. $B$ Proportion of patients with extravasation during treatment with and without 5-fluorouracil-cisplatin (5-FU-CDDP). The proportions of patients were compared using Fisher's exact test. AMR: Amrubicin; DTX: docetaxel; PTX: paclitaxel.

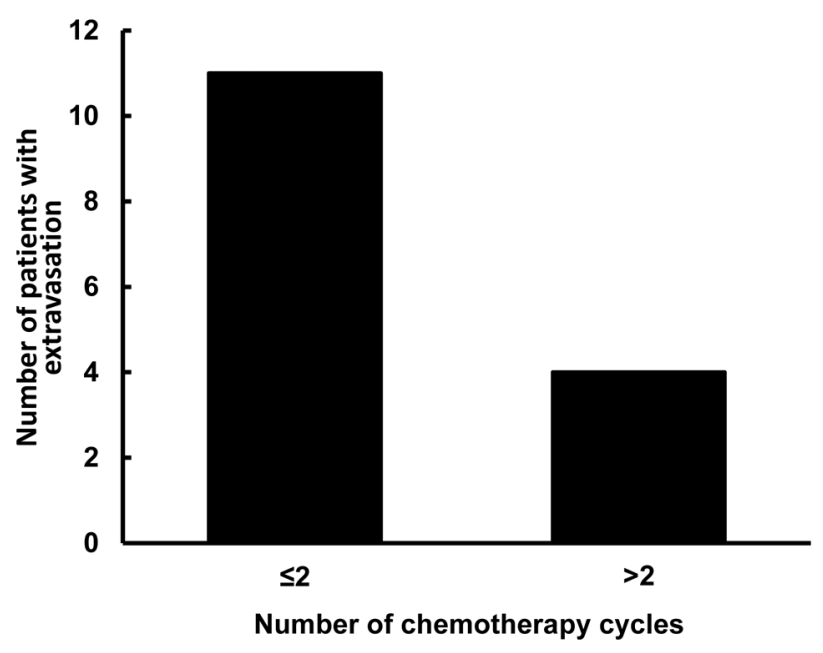

Figure 2. Number of patients with extravasation according to cycle of treatment with 5-fluorouracil-cisplatin.

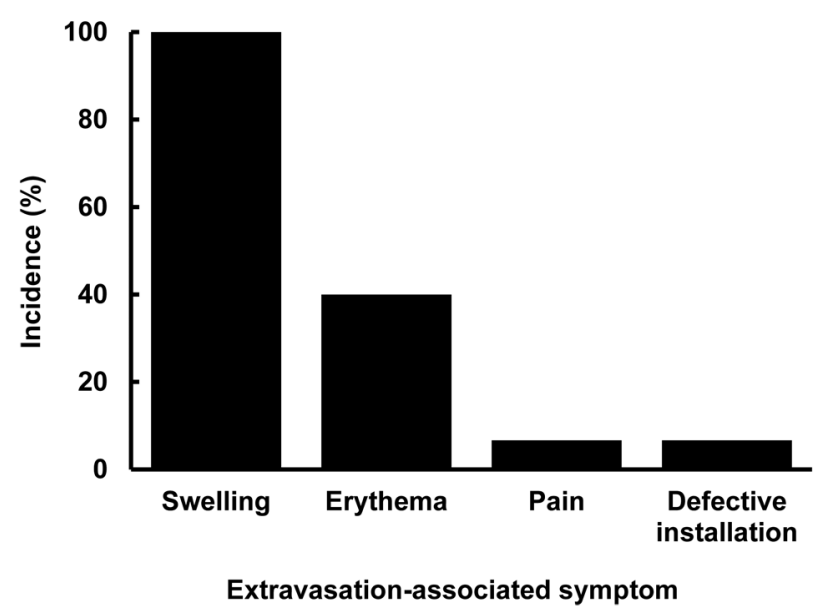

Figure 3. Incidence of extravasation-associated symptoms among patients undergoing treatment with 5-fluorouracil-cisplatin.

Table II. Characteristics of the chemotherapy regimen schedule at the study site.

\begin{tabular}{|c|c|c|c|c|c|}
\hline Regimen & Anticancer drug & Administration duration & Infusion dosage $(\mathrm{ml} / \mathrm{h})$ & Administration days & Interval \\
\hline \multirow[t]{2}{*}{ Combination } & Cisplatin & $2 \mathrm{~h}$ & 250 & Day 1 & 4 Weeks \\
\hline & 5-Fluorouracil & $24 \mathrm{~h}$ & 50 & Day 1-5 & \\
\hline \multirow[t]{2}{*}{ Combination } & Paclitaxel & $1 \mathrm{~h}$ & 250 & Day $1,8,15$ & 4 Weeks \\
\hline & Cisplatin & $2 \mathrm{~h}$ & 250 & Day $1,8,15$ & \\
\hline Monotherapy & Paclitaxel & $1 \mathrm{~h}$ & 250 & Day 1 & 1 Week \\
\hline Monotherapy & Docetaxel & $1 \mathrm{~h}$ & 250 & Day 1 & 3 Weeks \\
\hline Monotherapy & Amrubicin & $5 \mathrm{~min}$ & $500 *$ & Day $1,2,3$ & 3 Weeks \\
\hline
\end{tabular}

\footnotetext{
*Maximum infusion rate.
} 
These findings indicate the importance of suitable vascular access. Among patients with cancer treated using a central venous access device, the incidence of extravasation has been reported as $0.24 \%$ (5). The use of a central venous access device and a frequent change of the injection site in peripheral blood vessels may help prevent extravasation among patients treated with 5-FU-CDDP (3).

Extravasation requires immediate discontinuation of chemotherapy to treat symptoms that have already developed and to prevent further complications. Therefore, early detection of extravasation is paramount to good patient outcomes. In the present study, most cases of extravasation developed within the first two cycles of 5-FU-CDDP chemotherapy (Figure 2), which is within the early phase of treatment.

The most common symptom of extravasation was swelling, followed by erythema, which emerged in $40 \%$ of the patients (Figure 3). The side-effects of 5-FU include inflammation, painless erythema, and swelling at the extravasation site (6). In the present study, pain was rare among patients with extravasation associated with 5-FUCDDP treatment. Overall, these findings suggest that erythema and swelling may indicate extravasation, and patients should be carefully assessed for these symptoms, in particular, during the first two cycles of 5-FU-CDDP chemotherapy. In addition, awareness of these symptoms and their timing should be raised among both medical staff and patients undergoing 5-FU-CDDP chemotherapy.

Previous studies have reported that high flow pressure during infusion is one of the risk factors for extravasation during chemotherapy (7). However, in the present study, 14 of the extravasation cases $(63.6 \%)$ were treated at an infusion rate of $50 \mathrm{ml} / \mathrm{min}$, suggesting that treatment with 5-FU-CDDP is associated with a high incidence of extravasation independently of the infusion rate. Finally, a history of chemotherapy and extravasation did not increase the incidence of chemotherapy extravasation in this study (Table I).

This is the first study to report that the 5-FU-CDDP regimen remarkably increased the incidence of extravasation among patients undergoing chemotherapy. To prevent extravasation in this patient group, appropriate vascular access may be required. In addition, staff and patient awareness of the signs and symptoms of extravasation and shared vigilance are required to diagnose and treat extravasation in a timely manner, in particular, during the first two cycles of chemotherapy. Overall, these efforts can help reduce the rate of chemotherapy extravasation.

\section{Conflicts of Interest}

The Authors have no conflicts of interest to declare.

\section{Authors' Contributions}

Study conceptualisation: HT, YI, KY and TI. Study design: HT, YI, TI, HY and YK. Data collection: HT and IT. Data acquisition, analysis, and interpretation: HT, YI and YK. Article drafting: HT, YI and YK. Revising the draft critically for important intellectual content: HY. All Authors revised and approved the article.

\section{Acknowledgements}

The Authors would like to thank Editage (www.editage.com) for English language editing.

\section{References}

1 Kreidieh FY, Moukadem HA and El Saghir NS: Overview, prevention and management of chemotherapy extravasation. World J Clin Oncol 7(1): 87-97, 2016. PMID: 26862492. DOI: 10.5306/wjco.v7.i1.87

2 Jackson-Rose J, Del Monte J, Groman A, Dial LS, Atwell L, Graham J, O'Neil Semler R, O'Sullivan M, Truini-Pittman L, Cunningham TA, Roman-Fischetti L, Costantinou E, Rimkus C, Banavage AJ, Dietz B, Colussi CJ, Catania K, Wasko M, Schreffler KA, West C, Siefert ML and Rice RD: Chemotherapy extravasation: Establishing a national benchmark for incidence among cancer centers. Clin J Oncol Nurs 21(4): 438-445, 2017. PMID: 28738039. DOI: 10.1188/17.CJON.438-445

3 Ener RA, Meglathery SB and Styler M: Extravasation of systemic hemato-oncological therapies. Ann Oncol 15(6): 858862, 2004. PMID: 15151940. DOI: 10.1093/annonc/mdh214

4 Reddy SS, Somayaji S, Krishna Murthy M and Maka VV: 5Fluorouracil induced extravasation injury. Indian J Cancer 57(4): 467-469, 2020. PMID: 32769297. DOI: 10.4103/ijc.IJC_281_19

5 Narducci F, Jean-Laurent M, Boulanger L, El Bédoui S, Mallet Y, Houpeau JL, Hamdani A, Penel N and Fournier C: Totally implantable venous access port systems and risk factors for complications: A one-year prospective study in a cancer centre. Eur J Surg Oncol 37(10): 913-918, 2011. PMID: 21831566. DOI: $10.1016 /$ j.ejso.2011.06.016

6 St Luke's Cancer Alliance NHS, Chemotherapy Policies and Protocols [Internet]: Guidelines for Prevention and Management of Chemotherapy Extravasation. Available at: https:// www.royalsurrey.nhs.uk/download.cfm?doc=docm93jijm4n7168. pdf\&ver $=15074$ [Last accessed on December 7, 2020]

7 Pérez Fidalgo JA, García Fabregat L, Cervantes A, Margulies A, Vidall C, Roila F and ESMO Guidelines Working Group: Management of chemotherapy extravasation: ESMO-EONS clinical practice guidelines. Eur J Oncol Nurs 16(5): 528-534, 2012. PMID: 23304728. DOI: 10.1016/j.ejon.2012.09.004.

Received November 17, 2020

Revised December 8, 2020

Accepted December 10, 2020 